\title{
Mastering the infant donor eye: a new device for obtaining corneoscleral buttons for storage and corneal discs for keratoplasty
}

\author{
Israel N Nartey
}

\begin{abstract}
The design and use of a new device for obtaining corneoscleral buttons for storage and corneal discs for keratoplasty from neonatal and adult donor eyes is described. The device is simple to use and eliminates most of the difficulties in handling infant donor eyes. It thus minimises the risk of damage to the corneal endothelium and the formation of stretch striae, which reduce the quality of the donor material for transplantation.
\end{abstract}

The cornea of infant donors is difficult to handle because of its flexibility and the tendency for the tissue to fold over on itself. ${ }^{12}$ This, in addition to the lack of appropriate instruments for handling infant donor eyes during excision of corneoscleral buttons for storage, predisposes the endothelium to risk of damage. Because the infant donor eye may be too small to fit currently available commercial eye stands, it can be wrapped in gauze and hand held for corneal excision. This technique requires great skill, may be imprecise, and may lead to endothelial cell damage. $\mathrm{We}^{2}$ and other investigators ${ }^{34}$ have shown that this damage may be in the form of endothelial stretch striae or loss of the posterior membrane of the corneal endothelial cell, ${ }^{25}$ which reduces the quality of the donor material for transplantation.

Given the limitations of existing eye stands which can be modified, and the fact that an increasing number of surgeons now employ infant donor corneas for transplantation, ${ }^{6-8}$ a new device has been designed, produced, and tested with several donor eyes (age range 5 days-98 years) which can be used to hold the enucleated eye of full-term babies and adult donors for excision of corneoscleral buttons and corneal discs. A case is reported to show the value of the new device over the hand held technique of corneoscleral button excision.

\section{Description}

The device (Fig 1) comprises a base, main body, eye support block, and a clamping unit. The base consists of a solid circular plate $11.4 \mathrm{~cm}$ in

Moorfields Eye Bank, Moorfields Eye Hospital, and the Institute of Ophthalmology, London WC1H 9QS I N Nartey

Correspondence to: DrI N Nartey, Moorfields Ey Hospital, City Road, London ECIV 2PD.

Accepted for publication 2 August 1990 of a locating pin. The cylindrical head of the latter forms a fixed spindle by which the main body is mounted on to the base plate and is allowed to rotate.

The main body consists of a solid cylindrical base with a smooth surface. It is closed at the base except for a central aperture to fit over the locating pin. Projecting upwards from this base is an externally threaded, hollow cylinder, of a narrower diameter, defining a socket which houses the eye support block. A screw threaded radial hole extends into the base of the main body, from a point about $2 \mathrm{~mm}$ below its top edge, to receive a screw threaded locking pin. The locking pin has a head by which the pin may be rotated into engagement with the locating pin to lock the main body from rotation.

The eye support is a solid cylindrical block, hollowed out at its upper end. It has an axially extending threaded hole for accommodating the optic nerve stump of the donor eye. There are two interchangeable eye support blocks - a longer one for infant donor eyes and a shorter for use with adult donor eyes.

A threaded manipulating pin knurled at one end fits into the screw threaded hole in the eye support block and can be used to lift the latter out of the main body. When not in use, the manipulating pin can be stored in a blind storage hole at the side of the base plate.

A clamping unit for clamping the donor eye placed on the eye support comprises a lower cylindrical clamping sleeve and an upper clamping ring. The clamping sleeve is a hollow

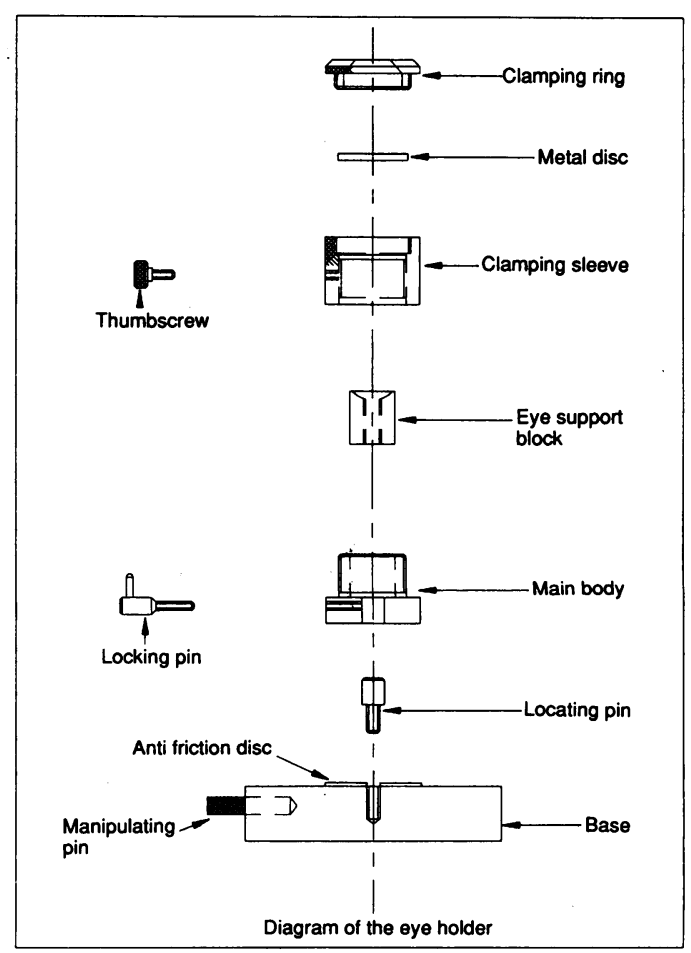

Figure 1 Diagram of the eye holder. 


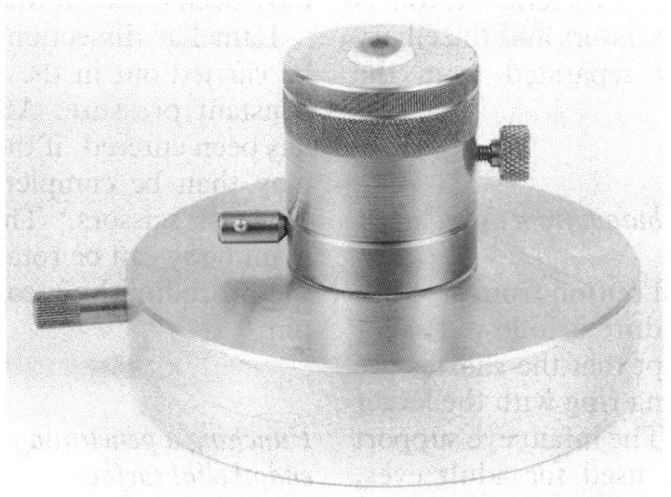

Figure 2 The device with an infant donor eye in situ.

cylinder, knurled at the upper third of its outer surface. It is divided internally into two unequal compartments by an annular ledge, above and below which are screw threads. The lower and deeper threads meet the external threads on the cylindrical projection of the main body, while the upper and shallower threads co-operate with external threads on the skirt of the clamping ring.

The latter is a broad ring with a central, circular aperture through which the cornea and a rim of sclera of the donor eye project as the clamping unit is screwed home on to the main body.

Two interchangeable clamping rings are provided; one with a small central aperture for infant donor eyes and another with a larger aperture for use in obtaining corneoscleral buttons from adults, though the clamping ring intended for infants may also be used for adult donor eyes. The clamping unit acts as an intraocular pressure adjuster which applies increasing compression to the donor eye as the clamping unit is screwed on to the main body.

A threaded thumbscrew fits into a threaded hole, half way up the side of the clamping sleeve, to lock the clamping unit to the main body projection.

With the clamping ring and eye support block removed, a metal disc may be placed in the upper compartment of the clamping sleeve, on the annular ledge, to act as a platform for a silicone rubber block for supporting a corneoscleral

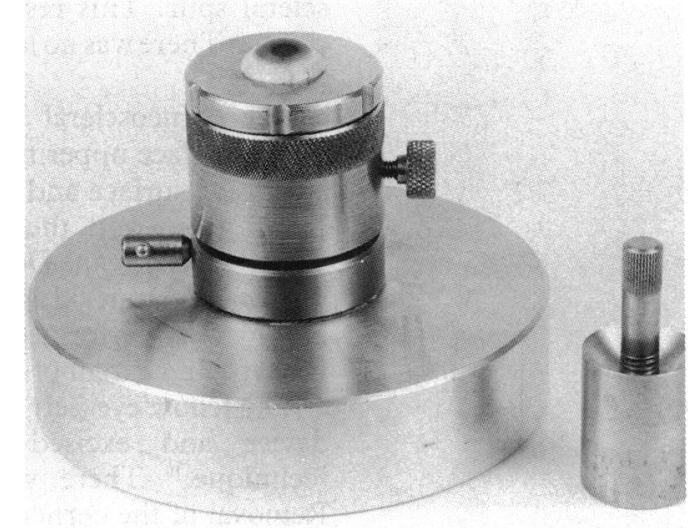

Figure 3 Adult donor eye in situ. Note replaced clamping ring, and infant eye support block with manipulating pin.

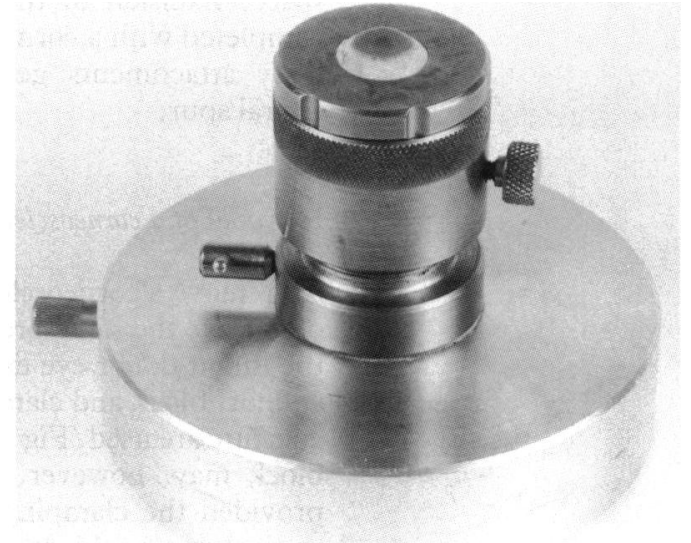

Figure 4 Adult donor eye supported in the infant block.

button to be punched out from the endothelial side of the button.

\section{Mode of use}

To prepare a corneoscleral button from an infant donor eye for storage

The main body, the infant eye support block, and the clamping sleeve are assembled on the base plate and the main body locked against rotation by the locking pin. The donor eye is cleansed and placed on the eye support with the optic nerve stump extending into the hole in the eye support block. The clamping ring is then screwed home and, provided the clamping sleeve has not engaged more than about half of the threads on the main body projection, the clamping ring will still be clear of the surface of the cornea.

By carefully screwing the clamping unit home clockwise with the thumbscrew, the cornea and a generous rim of sclera will present through the aperture in the clamping ring (Fig 2). When a suitable pressure is judged to have been reached, the thumbscrew is tightened to lock the clamping unit to the main body projection, and the locking pin is released. Rotating the assembly by the locking pin, with the eye engaged by a sharp blade in a holder, allows a simple and atraumatic incision to be made into the suprachoroidal

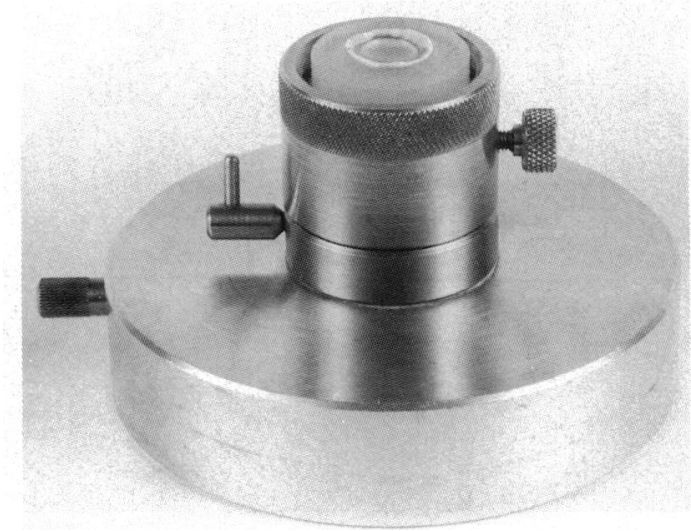

Figure 5 Set-up for punching a penetrating donor disc from the endothelial surface. 
space. Excision of the corneoscleral button is completed with a corneal scissors and the ciliary body attachments gently separated from the scleral spur.

\section{Removal of a corneoscleral button from the adult donor eye}

To remove a corneoscleral button from an adult donor eye the same procedure is followed as for the infant donor eye except that the shorter eye support block and clamping ring with the larger aperture are used (Fig 3). The infant eye support block may, however, be used for adult eyes, provided the clamping sleeve has not engaged more than two-thirds of the threads on the main body projection (Fig 4). If the clamping ring cannot be screwed home over the donor eye, the clamping sleeve may be unscrewed slightly to clear the surface of the cornea. The clamping ring is then screwed home. Which clamping ring is used for the adult donor eye depends on how large a corneoscleral button is required. The clamping ring with the larger aperture cannot, however, be used for the infant donor eye.

To cut a penetrating or lamellar disc from a donor eye The donor eye is placed in the same assembly as for obtaining a corneoscleral button. A forceps is used to centre the donor eye on the central axis of rotation, and, when a suitable tension for trepanation or lamellar dissection is judged to

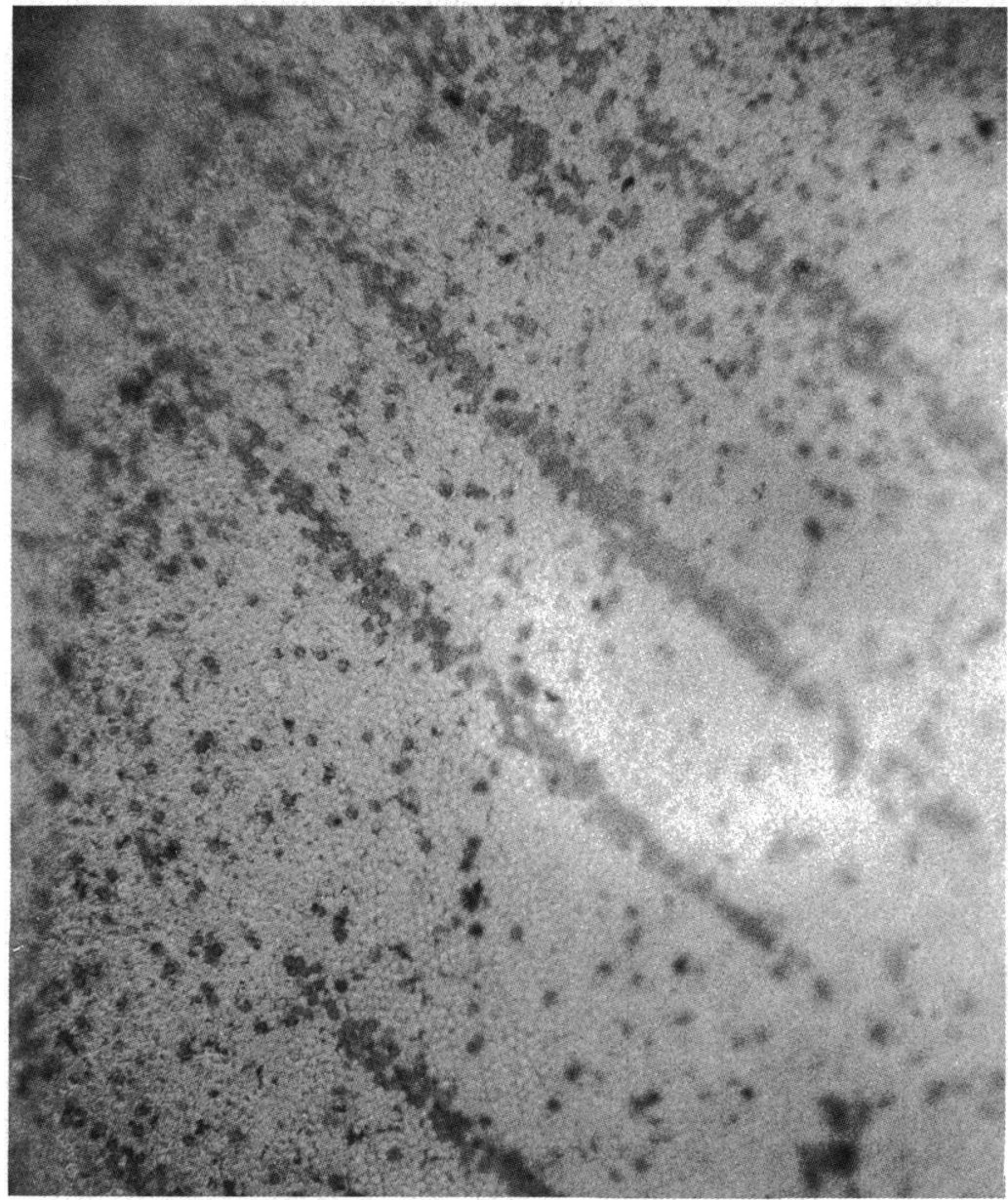

Figure 6 Right eye: hand held for corneoscleral button excision. Note multiple endothelial striae and numerous isolated damaged or dead cells with dark blue staining of the nuclei. (Fanus green/alizarin red $S$ stains, $\times 50$.) have been reached, the thumbscrew is tightened.

Lamellar dissection or trephination can then be carried out in the conventional manner at a constant pressure. After the anterior chamber has been entered, if the trephine is incomplete it may then be completed with a suitable sharp blade or scissors. ${ }^{9}$ The clamping unit and the main body can be rotated as a single unit during the procedure by means of the released locking pin.

\section{Punching a penetrating donor disc from the endothelial surface}

The main body and the clamping sleeve are assembled on the base plate without the eye support block and clamping ring, and the main body locked against rotation with the locking pin. The clamping sleeve is screwed home to the fullest extent on the main body projection and the thumbscrew tightened. The metal support disc is then placed in the upper compartment of the clamping sleeve so as to rest on the annular ledge. A silicone rubber block with a concave upper surface is then rested on the metal disc. The corneoscleral button is centred on the silicone block, endothelial surface uppermost (Fig 5), and punched out with a hand held trephine.

\section{Case report}

To show the value of the device in minimising stria formation in comparison with the hand held technique of corneal excision, a pair of donor eyes from a 3-week old baby was used as an example. The cause of death was respiratory failure, and the globes were enucleated $12 \mathrm{~h}$ post mortem after storage at $4^{\circ} \mathrm{C}$. The ocular history was normal for both eyes.

Each eye was examined $2 \mathrm{~h}$ after enucleation with the slit-lamp and the wide field specular microscope (the Pocklington, Keeler/Konan), particularly for striae and folds, before corneoscleral button excision, but none were found except for a haze of corneal oedema. Both eyes were rinsed in $0.9 \%$ normal saline and the conjunctiva was removed. The right eye was wrapped in gauze and hand held for corneoscleral button excision by the recommended standard technique. ${ }^{10}$ Some difficulty was encountered during initial incision into the suprachoroidal space and separation of the ciliary body from the scleral spur. This resulted in distortion of the cornea. There was no loss of the anterior chamber (AC).

The corneoscleral button was placed endothelial surface uppermost on a Teflon plate with a concave surface and stained immediately with Janus green and then with alizarin red $S$ as described by van Delft and colleagues ${ }^{11}$ and Taylor and Hunt. ${ }^{12}$ Light microscopy and photography were performed on the button to evaluate the type and extent of endothelial damage.

The fellow eye (left) was supported in the new device and excised by the recommended technique. ${ }^{10}$ There was no loss of the AC. Removal of the corneoscleral disc was relatively easy, as both hands were free to carry out the procedure with minimal distortion to the cornea. 


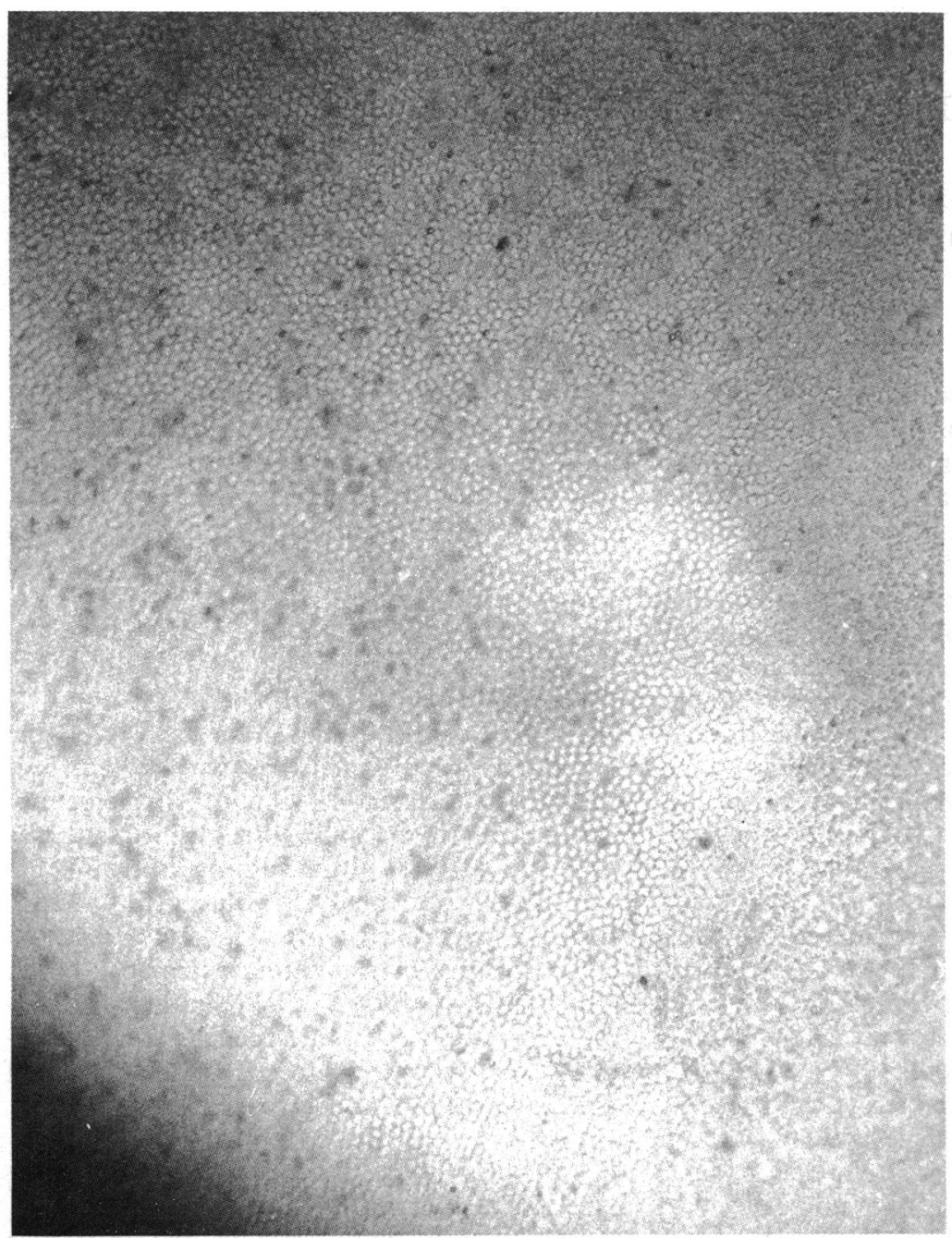

Figure 7 Left eye: supported in new device for corneoscleral button excision. Endothelial striae are absent. Staining of the intercellular borders with alizarin red $S$ shows the mosaic pattern of normal cells. Few isolated damaged cells are present. (Fanus green/alizarin red $S$ stains, $\times 50$.)

and photographed.

Light microscopy of the right button revealed multiple parallel lines of endothelial cells with darkly stained nuclei (Fig 6). In addition, there were areas of normal appearing endothelium interspersed with numerous isolated cells with darkly stained nuclei. Higher magnification from risk of damage. To this end, the author has devised an eye holder suitable for infant donors which leaves both hands free for the excision procedure in a controlled manner and allows better management of complications which may arise. It is believed that this innovation is an advance. The device is simple to use and has the added advantage that it can be used for adult donor eyes and corneal transplant procedures.

A comparative discussion of currently available eye stands is beyond the scope of this article. However, it is worth noting that the design of the device described here incorporates ideas from the Steele-Pierse eye stand, ${ }^{9}$ which is widely used, and provides good support for a wide range of donor eyes.

Janus green gives a more pronounced staining result and better contrast between non-vital and vital cells than trypan blue. ${ }^{13}$ The demarcation of non-vital from vital cell is further improved by counterstaining with alizarin red $\mathrm{S}$. Thus Janus green can replace trypan blue in combined trypan blue/alizarin red $S$ staining as shown by van Delft et al. ${ }^{11}$

Vital staining of the right cornea showed multiple striae at the level of the endothelium but none in the fellow cornea. It is tempting to conclude that the striae in the right cornea were the result of the handling difficulties encountered in the hand held excision technique. However, since no striae or folds were found at slit-lamp and specular microscopy, of both eyes, before corneoscleral button excision, it is reasonable to assume that indeed this is the case.

Although no striae were found in the left cornea, some stria formation is inevitable, even with the best technique. ${ }^{24}$ Care should therefore be taken during corneal excision to preserve the maximum number of viable endothelial cells for transplantation. showed that these lines corresponded to areas of endothelial cell damage or death as described elsewhere. ${ }^{2}$ The left button showed large areas of intact endothelium outlined by alizarin red $S$ and fewer isolated cells with darkly stained nuclei, but there were no striae (Fig 7).

\section{Discussion}

A number of surgeons do not transplant tissue from donors less than 3 years of age because of handling difficulties and the rapid change in corneal power during infancy, which decreases predictability.' Nevertheless, others use infant donor corneas for transplantation in aphakic patients. ${ }^{6-8}$

Damage to the endothelium during excision of corneoscleral buttons from infant donor eyes for storage and their subsequent use for transplantation has been quantified and discussed elsewhere. ${ }^{2}$ If the use of tissue from such donors for - transplants is to be encouraged, it would be desirable to have the appropriate instruments for their handling in order to protect the endothelium

I thank Mr Arthur D McG Steele for helpful suggestions and for reading the manuscript; Duncan Stamp and Paul Johnson for technical assistance; and Paul Grogan for the photographic processing. I am also grateful to Dr Emil Sherrard for encouragement.

1 Wilson SE, Bourne WM. Corneal preservation. Surv Ophthalmol 1989; 33: 237-59.

2 Narteý IN, Sherrard ES, Steele ADMcG. Manipulative damage to the endothelium of infant and adult donor corneas. Br F Ophthalmol 1990; 74: 261-4.

3 Roozitalab MH, Requard JJ, Fogle JA, Green WR, Graham CR Jr. Endothelial stretch striae in donor corneas: cause, CR Jr. Endothelial stretch striae in donor corneas: cause,
significance, and prevention. Md Med $\mathcal{F} 1979 ; 28: 47-55$.

4 Grutzmacher RD, Oiland D, Mckillop BR, Bunt-Milam AH. Donor corneal endothelial striae. Am $\mathcal{F}$ Ophthalmol 1986; 102: 508-15.

5 Polack FM. Penetrating keratoplasty. In: Polack FM, ed. Corneal transplantation. New York: Grune and Stratton, 1977: 135-46.

6 Pfister RR, Breaud S. Aphakic refractive penetrating keratoplasty using newborn donor corneas. Ophthalmology 1983; 90: 1207-12.

7 Wood TO, Nissernkorn L. Infant donor corneas for penetrating keratoplasty. Ophthalmic Surg 1981; 12: 500-2.

8 Fong LP, Gladstone D, Casey TA. Corneoscleral rim cultures: donor contamination. A case of fungal endophthalmitis donor contamination. A case of fungal endopht
transmitted by K-sol cornea. Eye $1988 ; 2: 670-6$.

9 transmitted by K-sol cornea. Eye $1988 ; 2: 670-6$. device for obtaining corneal discs. Br f Ophthalmol 1978; 62: 86-8.

10 Krachmer JH Jr. Medical Standards of the Eye Bank Association of America. 1984: section 8:1

11 van Delft J, Oosterhuis J, Barthen E, Eijkelenboom A. Janus green vital staining of the cornea. Doc Ophthalmol 1983; 55: $47-50$.

12 Taylor $M J$, Hunt CJ. Dual staining of corneal endothelium with trypan blue and alizarin red S: importance of $\mathrm{pH}$ for the dye-lake reaction. Brf Ophthalmol 1981; 65: 815-9.

13 Hartman C, Rieck P. A new test for endothelial viability. The Janus green photometry technique. Arch Ophthalmol 1989; 107: 1511-5. 